\title{
Influence of orotracheal intubation, shock and acute renal failure of elderly patients admitted to ICU
}

\author{
JE Romo Gonzales*, J Silva Obregón', C Martin Dal Gesso', P Gallardo Culebradas², S Saboya Sanchez², \\ M Torralba ${ }^{4}$
}

From ESICM LIVES 2015

Berlin, Germany. 3-7 October 2015

\section{Objectives}

Describe the clinic-epidemiological characteristics of elderly patients ( ${ }^{3} 80$ years) admitted to ICU for medical reasons, analyze the impact of orotracheal intubation (IOT), acute renal failure $(\mathrm{RF})$ and shock at the mortality adjusted for habitual scales (APACHE II, SAPS II and SOFA).

\section{Methods}

Retrospective cohort study from January-2003 to May2011. In this study we included patients $\left({ }^{3} 80\right.$ years $)$ admitted to the ICU for medical causes, except ischemic heart disease or arrhythmias. Logistic regressions were used to calculate the OR of different variables as predictors of mortality (dependent variable). The ability to predict mortality of different "scores" adjusted for IOT, RF and Shock was performed using multivariate logistic regressions.

\section{Results}

They were analyzed 95 patients, $58 \%$ male; median age was 81 years (IQR $80-83$ years). 52 patients died (54.7\%), withdrawing life-sustaining therapy in 22 patients $(23.2 \%)$. They were readmitted to ICU $3.3 \%$. The median number of days in the ICU and hospital were 4(IQR: 1.4 to 11 ) and 12 days (IQR: 6-25) respectively. The median APACHE II, SAPS II and SOFA were 23 (IQR 18-29), 7 (IQR: 4-10) and 49 (IQR: 40-65). $63 \%$ was intubated (60/95) for a median of 4 days (IQR: $1-13.75)$, died $73.3 \%(44 / 60) .65 .3 \%$ presented shock (62/ 95), 66.1\% died (41/62). 61\% renal failure (58/95) and $60.3 \%$ died $(35 / 58)$.

${ }^{1}$ Hospital Universitario de Guadalajara, Intensive Care Unit, Guadalajara, Spain Full list of author information is available at the end of the article
Mortality was associated in univariate analysis with IOT (OR: 9.3; IC95\% 3.5-24.6; p < 0.0001), Shock (OR: 3.9; IC95\% 1.6-9.6; $\mathrm{p}=0.002)$ ), but not with FR (OR 1.8; IC95\% 0.8-4.1; $\mathrm{p}=0.17)$ or age $(\mathrm{p}=0.65)$; for every one point increase in score of APACHE II, SAPS II and SOFA, the increased in mortality was $16 \%, 32 \%$ and $13 \%$ respectively ( $\mathrm{p}<0.0001$ in all three analyzes). In a conditional stepwise multivariate analysis, adjusting for FR, IOT, shock, age, sex and different "scores", only the SAPS II (OR 1.12, 95\% CI 1.6-1.18; $\mathrm{p}<0.0001$ ) and IOT (OR 3.6, 95\% CI 1.12-11.37; $\mathrm{p}=0.031$ ) were independent predictors of mortality.

\section{Conclusions}

In our series, the overall mortality of elderly patients $\left({ }^{3} 80\right.$ years) admitted to the ICU was very high (54.7\%). The orotracheal intubation and SAPS II are independent variables associated to mortality patients admitted to ICU for medical cause with at least 80 years.

\section{Authors' details}

${ }^{1}$ Hospital Universitario de Guadalajara, Intensive Care Unit, Guadalajara, Spain. ${ }^{2}$ Hospital Universitario de Guadalajara, Anaesthesiology, Guadalajara, Spain. ${ }^{3}$ Hospital Universitario Puerta de Hierro Majadahonda, Intensive Care Unit, Madrid, Spain. ${ }^{4}$ Hospital Universitario de Guadalajara, Internal Medicine, Guadalajara, Spain.

Published: 1 October 2015

doi:10.1186/2197-425X-3-S1-A658

Cite this article as: Romo Gonzales et al:: Influence of orotracheal intubation, shock and acute renal failure of elderly patients admitted to ICU. Intensive Care Medicine Experimental 2015 3(Suppl 1):A658.

\section{SpringerOpen ${ }^{\circ}$}

(C) 2015 Romo Gonzales et al.; This is an Open Access article distributed under the terms of the Creative Commons Attribution License (http://creativecommons.org/licenses/by/4.0), which permits unrestricted use, distribution, and reproduction in any medium, provided the original work is properly cited. 